\title{
Article \\ Variation of Autonomic Nervous System Function by Age and Gender in Thai Ischemic Stroke Patients
}

\author{
Warawoot Chuangchai $^{1}{ }^{\mathbb{D}}$, Wiraporn Pothisiri ${ }^{1, *}$ and Phumdecha Chanbenjapipu ${ }^{2}$ \\ 1 College of Population Studies, Chulalongkorn University, Bangkok 10330, Thailand; warawoot.c@chula.ac.th \\ 2 Faculty of Nursing, Siam University, Bangkok 10160, Thailand; phumdecha.cha@siam.edu \\ * Correspondence: wiraporn.p@chula.ac.th; Tel.: +66-0-2218-7346; Fax: +66-0-2255-1469
}

check for updates

Citation: Chuangchai, W.; Pothisiri, W.; Chanbenjapipu, P. Variation of Autonomic Nervous System Function by Age and Gender in Thai Ischemic Stroke Patients. Brain Sci. 2021, 11, 380. https://doi.org/10.3390/ brainsci11030380

Academic Editor: Jan Brogger

Received: 27 January 2021

Accepted: 15 March 2021

Published: 17 March 2021

Publisher's Note: MDPI stays neutral with regard to jurisdictional claims in published maps and institutional affiliations.

Copyright: (c) 2021 by the authors. Licensee MDPI, Basel, Switzerland. This article is an open access article distributed under the terms and conditions of the Creative Commons Attribution (CC BY) license (https:/ / creativecommons.org/licenses/by/ $4.0 /)$.

\begin{abstract}
Background: Ischemic stroke is one of the major causes of disability and mortality. Its effects on the autonomic nervous system (ANS) through nonlinear heart rate variability (HRV) and pulse transit time (PTT) have not been well explored among Thai patients. Objective: This study aims to demonstrate the association between ANS and ischemic stroke through nonlinear HRV and PTT. Methods: In total, 111 patients were enrolled in the study and their short-term HRV and PTT data were collected. Results: Parasympathetic tone was higher in elderly patients ( $\geq 60$ years). The elderly patients had a higher SD1 but lower SD2 and SD2/SD1 than the younger patients, and a similar pattern was found in the female patients compared to the male patients. These findings were supported by the results of the Poincaré plots. Older and female patients had circular plots and approximately round plots, respectively. Moreover, the parasympathetic nervous system (PNS) response was moderate and positively associated with SD1 $(r=0.47, p<0.001)$ and PTT $(r=0.29$, $p=0.002)$, and negatively associated with SD2 and SD2/SD1 $(r=-0.47, p<0.001)$, after controlling for age and sex. Conclusions: The PNS response was predominant in older and female patients whereas the sympathetic response was lower than in the younger and male patients, which reflected certain characteristics of ANS response to ischemic stroke. Moreover, nonlinear parameters of SD1, SD2, SD2/SD1, and Poincaré plots including PTT are useful and recommended in investigating ANS, particularly in PNS, among ischemic stroke patients.
\end{abstract}

Keywords: autonomic nervous system; ischemic stroke; heart rate variability; pulse transit time

\section{Introduction}

Ischemic stroke is the most common type of stroke across the globe and is one of the serious causes of disability and mortality in Thailand [1]. Aging is the greatest risk factor, followed by male gender, within the Thai population [2]. An ischemic stroke is a neurovascular event characterized by the interruption of the blood supply within the brain [3]. It is closely associated with autonomic nervous system (ANS) dysfunction and cardiovascular responses, which likely result in the development of severe ischemic events [4].

Both the parasympathetic nervous system (PNS) and sympathetic nervous system (SNS) are branches of the ANS, which controls almost all visceral, vascular, and metabolic functions [3]. Heart rate variability (HRV) data have been demonstrated to reflect the activity of the ANS. HRV is a noninvasive measurement recorded via an electrocardiogram (ECG). HRV reflects variations between consecutive inter-beat-intervals as R-R (RR) intervals [5]. In terms of the time-domain parameters, previous studies have indicated the mean RR interval and the root mean square of successive RR interval differences (RMSSD) to positively associate with the PNS response. Meanwhile, the mean heart rate (HR) and Baevsky's stress index have been shown to link with the SNS response [6,7]. Low frequency (LF), high frequency $(\mathrm{HF})$, and the ratio of LF to HF power (LF/HF) are broadly used as the frequency domain parameters. Previous studies have indicated that LF and HF reflect SNS and PNS responses, respectively, whereas the LF/HF ratio reflects the sympathovagal balance $[8,9]$. 
In addition, nonlinear HRV parameters are determined by complex interactions among hemodynamic, electrophysiological, and humoral variables, as well as by autonomic and central nervous system regulations [10]. The parameters of the standard deviation (SD) SD1, SD2, and SD2/SD1 have been used for assessing ANS modulation. Previous studies have indicated that SD1 was likely linked to the PNS, whereas SD2 and SD2/SD1 were likely linked to the SNS $[8,11]$. Moreover, pulse transit time (PTT) is a measurement of duration between pulse peaks obtained by photoplethysmography (PPG) and ECG sensors. A previous study indicated that PTT was associated with arterial stiffness and blood pressure, which reflected vascular tone [12]. However, the nonlinear parameters and PTT in ischemic stroke patients have produced uncertain results and remain to be clarified.

Within the Thai population, the study of ischemic stroke using HRV data, particularly nonlinear HRV, is limited. Only a few studies indicated nonlinear parameters [13-15]. Recently, a study of HRV in chronic ischemic stroke patients was carried out with a small sample of 13 patients [16]. Numerous HRV studies have compared ischemic stroke patients with non-patients, exploring whether this association varies by age and sex [17-20]. There has been no study that has investigated the associations between ANS and age and sex among ischemic stroke patients. If the negative effects of ischemic stroke are to be reduced, then the underlying mechanisms of functional impairment need to be investigated. Therefore, this study aimed to investigate the ANS response and its association with age and sex among ischemic stroke patients. Nonlinear HRV parameters, including SD1, SD2, SD2/SD1, and Poincaré plots including PTT, were used. The present study will provide results which help to better understand ANS changes in patients with ischemic stroke.

\section{Materials and Methods}

\subsection{Participants}

In this cross-sectional study, patients who admitted as a new case with the first-ever stroke were observed from the stroke unit of Thammasat University Hospital (TUH), Thailand. All potentially eligible patients were screened using magnetic resonance imaging (MRI). Inclusion criteria were sex (both males and females), age (18 years or older), a diagnosis of ischemic stroke, and an early mobilization within $24 \mathrm{~h}$ of stroke onset. Exclusion criteria were a diagnosis with hemorrhagic stroke, valvular heart disease, or atrial fibrillation.

Among them, three patients were unqualified to enroll in the study. As a result, a total of 111 ischemic stroke patients were included at the final stage. They ranged in age from 23 to 85 years and included 63 males (30-83 years) and 48 females (23-85 years), as shown in Figure 1. Moreover, all patients in the study were diagnosed with right middle cerebral artery (R-MCA) infarction, except one that had left MCA infarction. No insular lesions were detected.

\subsection{Measurements}

HRV data were collected during the acute phase with a short-term, 5-min ECG recording lead II protocol with fingertip PPG from resting patients, breathing normally, in a supine position. The parameters obtained for the HRV analysis included mean RR, mean HR, RMSSD, stress index, LF, HF, LF/HF ratio, SD1, SD2, and SD2/SD1, whereas the PPG data were collected for the PTT analysis. The ECG measurements were performed in the morning between 6 and 7 AM. The room was maintained at a temperature of $25^{\circ} \mathrm{C}$. The ECG measurements were taken with a sampling rate of $1000 \mathrm{~Hz}$ using the PowerLab 26T data acquisition system (ADInstruments, Sydney, Australia) and recorded with LabChart software. In addition, blood pressure (BP) was assessed using an automatic blood pressure monitor (IntelliVue MP40, Philips, Amsterdam, The Netherlands). Systolic blood pressure (SBP) and diastolic blood pressure (DBP) were collected for pulse pressure (PP) and mean arterial pressure (MAP) [21]. 
Protocol

Inclusion

Exclusion

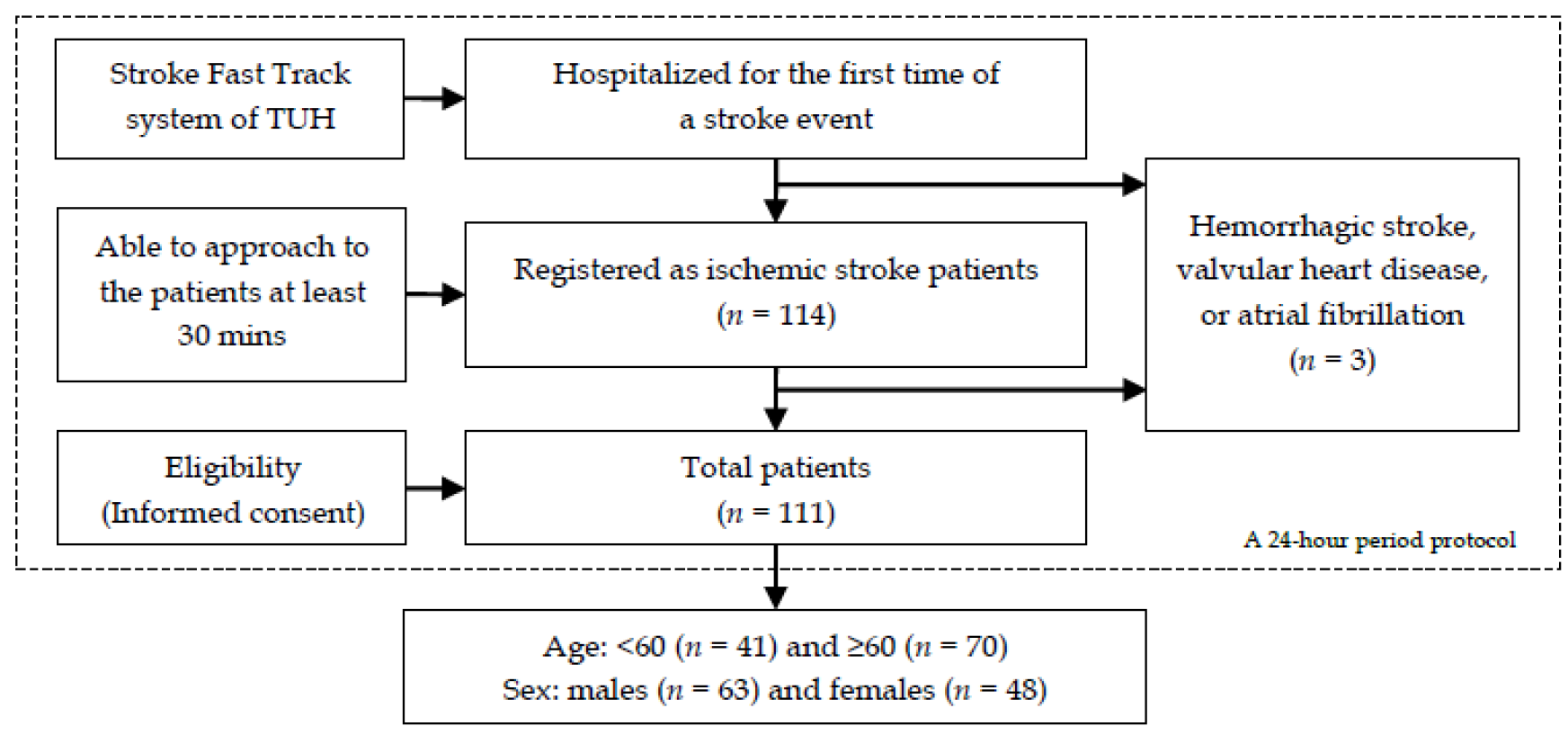

Figure 1. Flow diagram defining the study patients with a first ischemic stroke.

\subsection{Statistical Analysis}

All HRV data were analyzed using Kubios HRV Standard software [22], version 3.3.1. Mean RR, RMSSD, and SD1 parameters were computed for the PNS index, whereas mean HR, stress index, and SD2 parameters were computed for the SNS. Levels of parasympathetic and sympathetic tones were categorized as low (lower than -2.01 ), relatively low ( -2.00 to -1.01$)$, average ( -1.00 to 1.00 ), relatively high (1.01 to 2.00$)$, and high (higher than 2.01). LF, HF and LF/HF ratio were computed in normalized unit (n.u.). Besides, the PTT was calculated based on time differences between peaks of PPG waves and R waves: PPG peak $-\mathrm{R}$ peak/1000. The PP was computed as SBP-DBP, and the MAP was obtained as DBP $+1 / 3$ of PP. A normality test (Shapiro-Wilk) was applied to all collected values. Results were presented as mean \pm standard deviation, number $(\%)$, or median and interquartile range $\left(25-75^{\circ}\right)$. Differences between groups were compared with an independent (unpaired) $t$-test for normal distribution and the Mann-Whitney $U$ test for non-normal distribution. Relationships were analyzed with the nonparametric partial correlation while controlling for age and sex. A level of significance was set at $p<0.05$ (two-tailed). All statistical analyses were performed with IBM SPSS Statistics version 22. Effect sizes were estimated and interpreted with Cohen's $d$, eta-squared $\left(\eta^{2}\right)$, or correlation $(r)$ [23].

\section{Results}

The present study included patients aged $61.57 \pm 12.64$ years, with 63 males $(56.80 \%)$ and 48 females $(43.20 \%)$. The PNS and SNS indices were $0.18 \pm 2.00$ and $1.36 \pm 1.91$, respectively. LF was lower than HF with the ratio of $1.45 \pm 1.79$. Additionally, SD1 was lower than SD2 with an SD2/SD1 of $1.47 \pm 0.62$. PTT, SBP, DBP, PP and MAP values were $0.24 \pm 0.03,149.01 \pm 20.03,82.95 \pm 13.40,66.05 \pm 17.46$, and $104.97 \pm 13.63$, respectively, as shown in Table 1. 
Table 1. Means and standardization of all indices, ischemic stroke patients $(n=111)$.

\begin{tabular}{cc}
\hline Variable (Unit) & Total Patients \\
\hline Age (years) & $61.57 \pm 12.64$ \\
Male (\%) & $56.80 \%$ \\
\hline Autonomic indices & $0.18 \pm 2.00$ \\
PNS index & $1.36 \pm 1.91$ \\
SNS index & \\
HRV indices & $46.63 \pm 20.82$ \\
LF (n.u.) & $52.85 \pm 20.47$ \\
HF (n.u.) & $1.45 \pm 1.79$ \\
LF/HF ratio & $42.80 \pm 9.48$ \\
SD1 (\%) & $57.17 \pm 9.48$ \\
SD2 (\%) & $1.47 \pm 0.62$ \\
SD2/SD1 & \\
PTT and BP indices & $0.24 \pm 0.03$ \\
PTT (ms) & $149.01 \pm 20.03$ \\
SBP (mmHg) & $82.95 \pm 13.40$ \\
DBP (mmHg) & $66.05 \pm 17.46$ \\
PP (mmHg) & $104.97 \pm 13.63$ \\
MAP (mmHg) &
\end{tabular}

The sample patients were categorized by age as under or over 60 years, with 41 young patients aged $48.46 \pm 8.59$ years ( 24 males aged $49.17 \pm 7.60$ years and 17 females aged $47.47 \pm 9.98$ years), and 70 elderly patients aged $69.24 \pm 7.07$ years (39 males aged $70.00 \pm 7.30$ years and 31 females aged $68.29 \pm 6.77$ years). In addition, patients were categorized by sex, with 63 male patients aged $62.06 \pm 12.58$ years and 48 female patients aged $60.92 \pm 12.82$ years.

Moreover, the young patients were associated with PNS index of $-0.26 \pm 1.45$ and SNS index of $1.57 \pm 1.97$, while the older patients were associated with PNS index of $0.44 \pm 2.22$ and SNS index of $1.24 \pm 1.87$. Besides, the male patients were associated with PNS index of $0.07 \pm 1.86$ and SNS index of $1.45 \pm 1.94$, while the female patients were associated with PNS index of $0.33 \pm 2.17$ and SNS index of $1.25 \pm 1.89$.

By comparing the young and older patients as shown in Table 2, there were statistically significant differences between the groups by age $(p<0.001)$, PP $(p=0.004)$, SD2/SD1 $(p=0.014), \mathrm{SD} 2(p=0.023), \mathrm{SD} 1(p=0.024)$, parasympathetic tone $(p=0.030)$, and DBP $(p=0.041)$. In addition, there was a large effect by age $\left(\eta^{2}=0.69\right)$, relatively large effects in SD2 $(d=0.45)$ and SD1 $(d=-0.45)$, medium effects in PP $\left(\eta^{2}=0.08\right)$ and SD2/SD1 $\left(\eta^{2}=0.06\right)$, and small effects in parasympathetic tone $\left(\eta^{2}=0.04\right)$ and DBP $\left(\eta^{2}=0.04\right)$. In contrast, no significant differences were found between the groups in sympathetic tone, LF, HF, LF/HF ratio, PTT, SBP, and MAP.

By comparing the male and female patients as shown in Table 2 , statistically significant differences were found between the groups in SD2/SD1 $(p=0.014)$, SD2 $(p=0.023)$, SD1 $(p=0.026)$, and LF $(p=0.048)$. Moreover, relatively large effects were found in SD2 $(d=0.44)$, SD1 $(d=-0.43)$, and LF $(d=0.38)$, and a small effect in SD2/SD1 $\left(\eta^{2}=0.05\right)$. In contrast, no significant differences were found between the groups in terms of age, autonomic tones, HF, LF/HF ratio, and PTT and BP indices. 
Table 2. Descriptive statistics and comparison results by age and sex in ischemic stroke patients.

\begin{tabular}{|c|c|c|c|c|c|c|}
\hline Variable (Unit) & Age $<60(n=41)$ & Age $\geq 60(n=70)$ & $p$ Value & Males $(n=63)$ & Females $(n=48)$ & $p$ Value \\
\hline Age (years) & $\begin{array}{c}49.00 \\
(45.00-56.00)\end{array}$ & $\begin{array}{c}69.00 \\
(62.00-76.00)\end{array}$ & $<0.001^{b}$ & $62.06 \pm 12.58$ & $60.92 \pm 12.82$ & 0.638 \\
\hline \multicolumn{7}{|l|}{ Autonomic tones } \\
\hline Parasympathetic tone & & & $0.030^{\mathrm{b}}$ & & & 0.826 \\
\hline Low & $3(7.30 \%)$ & $1(1.40 \%)$ & & $2(3.20 \%)$ & $2(4.20 \%)$ & \\
\hline Relatively low & $11(26.80 \%)$ & $13(18.60 \%)$ & & $13(20.60 \%)$ & $11(22.90 \%)$ & \\
\hline Average & $21(51.20 \%)$ & $36(51.40 \%)$ & & $33(52.40 \%)$ & $24(50.00 \%)$ & \\
\hline Relatively high & $3(7.30 \%)$ & $7(10.00 \%)$ & & $7(11.10 \%)$ & $3(6.30 \%)$ & \\
\hline High & $3(7.30 \%)$ & $13(18.60 \%)$ & & $8(12.70 \%)$ & $816.70(\%)$ & \\
\hline Sympathetic tone & & & 0.281 & & & 0.905 \\
\hline Low & 0 & $11.40(\%)$ & & 0 & $1(2.10 \%)$ & \\
\hline Relatively low & $2(4.90 \%)$ & $6(8.60 \%)$ & & $5(7.90 \%)$ & $3(6.30 \%)$ & \\
\hline Average & $17(41.50 \%)$ & $28(40.00 \%)$ & & $25(39.70 \%)$ & $20(41.70 \%)$ & \\
\hline Relatively high & $6(14.60 \%)$ & $17(24.30 \%)$ & & $14(22.20 \%)$ & $9(18.80 \%)$ & \\
\hline High & $1639.00(\%)$ & $1825.70(\%)$ & & $19(30.20 \%)$ & $15(31.30 \%)$ & \\
\hline \multicolumn{7}{|l|}{ HRV indices } \\
\hline LF (\%) & $43.00 \pm 16.99$ & $37.67 \pm 16.22$ & 0.104 & $42.35 \pm 16.65$ & $36.08 \pm 16.09$ & $0.048^{\mathrm{a}}$ \\
\hline HF (\%) & $43.74 \pm 20.70$ & $49.88 \pm 21.37$ & 0.142 & $44.94 \pm 19.95$ & $51.11 \pm 22.55$ & 0.130 \\
\hline $\mathrm{LF} / \mathrm{HF}$ ratio & $1.16(0.45-1.74)$ & $0.86(0.42-1.38)$ & 0.167 & $1.16(0.48-1.65)$ & $0.70(0.40-1.32)$ & 0.106 \\
\hline SD1 (\%) & $40.17 \pm 8.97$ & $44.35 \pm 9.49$ & $0.024^{\mathrm{a}}$ & $41.07 \pm 8.34$ & $45.08 \pm 10.45$ & $0.026^{\mathrm{a}}$ \\
\hline SD2 (\%) & $59.83 \pm 8.97$ & $55.61 \pm 9.49$ & $0.023^{a}$ & $58.95 \pm 8.33$ & $54.84 \pm 10.45$ & $0.023^{a}$ \\
\hline SD2/SD1 & $1.55(1.11-1.91)$ & $1.26(0.95-1.59)$ & $0.014^{b}$ & $1.50(1.15-1.85)$ & $1.14(0.94-1.59)$ & $0.014^{b}$ \\
\hline \multicolumn{7}{|l|}{ PTT and BP indices } \\
\hline PTT $(\mathrm{ms})$ & $0.24(0.22-0.26)$ & $0.24(0.22-0.26)$ & 0.971 & $0.24(0.22-0.26)$ & $0.24(0.22-0.25)$ & 0.520 \\
\hline $\mathrm{SBP}(\mathrm{mmHg})$ & $\begin{array}{c}149.00 \\
(129.00-158.00)\end{array}$ & $\begin{array}{c}150.00 \\
(139.00-159.00)\end{array}$ & 0.308 & $\begin{array}{c}149.00 \\
(137.00-156.00)\end{array}$ & $\begin{array}{c}150.00 \\
(138.25-159.75)\end{array}$ & 0.555 \\
\hline $\mathrm{DBP}(\mathrm{mmHg})$ & $\begin{array}{c}83.00 \\
(79.50-92.00)\end{array}$ & $\begin{array}{c}82.00 \\
(75.00-86.50)\end{array}$ & $0.041^{\mathrm{b}}$ & $\begin{array}{c}83.00 \\
(77.00-90.00)\end{array}$ & $\begin{array}{c}82.50 \\
(76.25-89.50)\end{array}$ & 0.931 \\
\hline PP (mmHg) & $\begin{array}{c}60.00 \\
(48.50-67.00)\end{array}$ & $\begin{array}{c}67.00 \\
(58.75-78.00)\end{array}$ & $0.004^{b}$ & $\begin{array}{c}67.00 \\
(56.00-72.00)\end{array}$ & $\begin{array}{c}67.00 \\
(55.50-74.75)\end{array}$ & 0.903 \\
\hline MAP (mmHg) & $106.71 \pm 14.84$ & $103.96 \pm 12.87$ & 0.307 & $104.41 \pm 12.95$ & $105.71 \pm 14.57$ & 0.622 \\
\hline
\end{tabular}

Note: Results were presented as median and interquartile ranges $\left(25-75^{\circ}\right)$ or mean \pm standard deviation or number (\%). Significant at $p<0.05$ with ${ }^{\mathrm{a}}$ independent $t$-test or ${ }^{\mathrm{b}}$ Mann-Whitney $U$ test.

As shown in Figure 2, patients under 60 years old mostly showed elliptical plots where the length of the SD1 line was shorter than the SD2 line. By contrast, patients over 60 years old mostly showed circular plots where the SD1 line was slightly shorter than the SD2 line. Male patients mostly showed oval-shaped plots, similar to patients under 60 years old. Conversely, female patients mostly showed approximately round plots where the SD1 line was nearly equal to the SD2 line. 
A

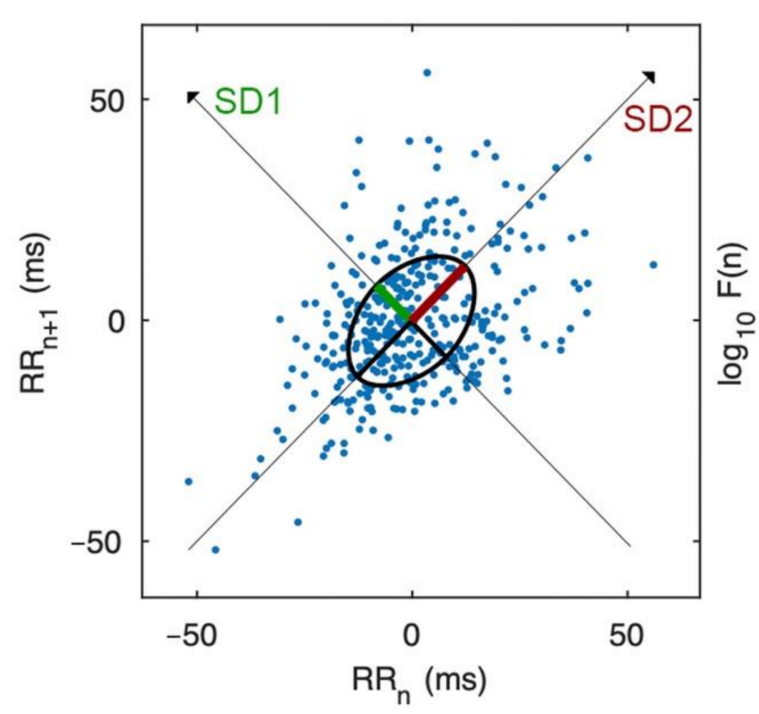

Age $<60(n=41)$

Proportion: SD1 <SD2

Shape: Ellipse

C

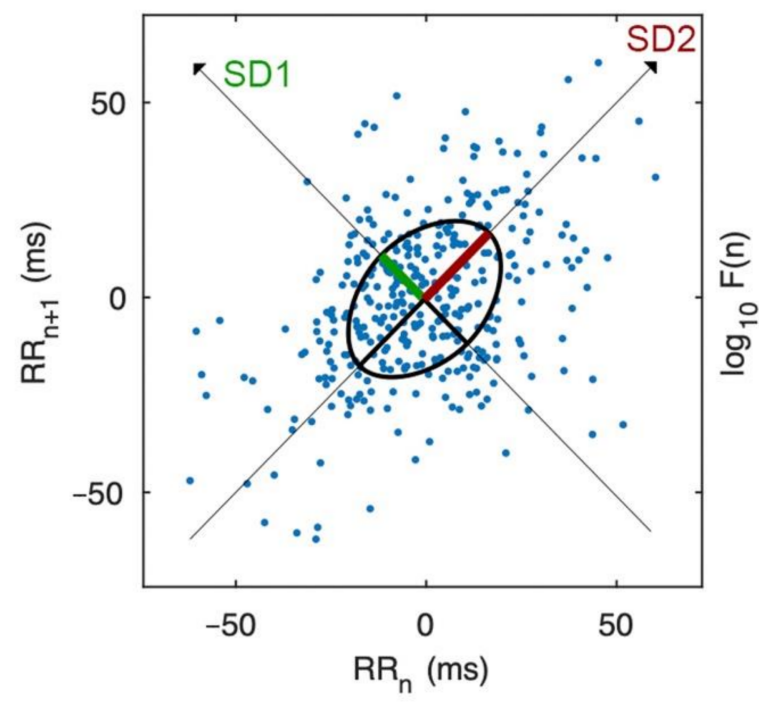

Males $(n=63)$

Proportion: SD1 < SD2

Shape: Oval
B

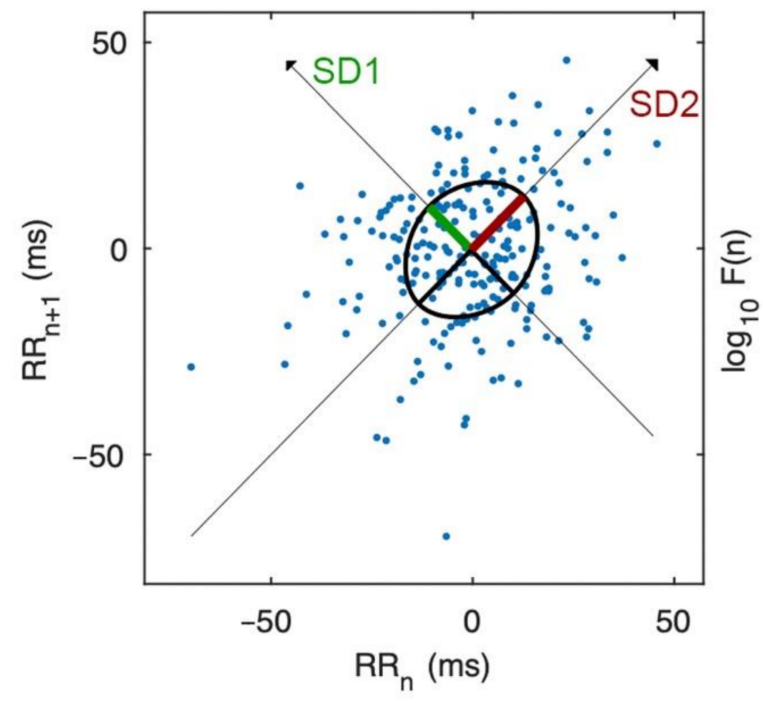

Age $\geq 60(n=70)$

Proportion: SD1 $\leq$ SD2

Shape: Circle

D

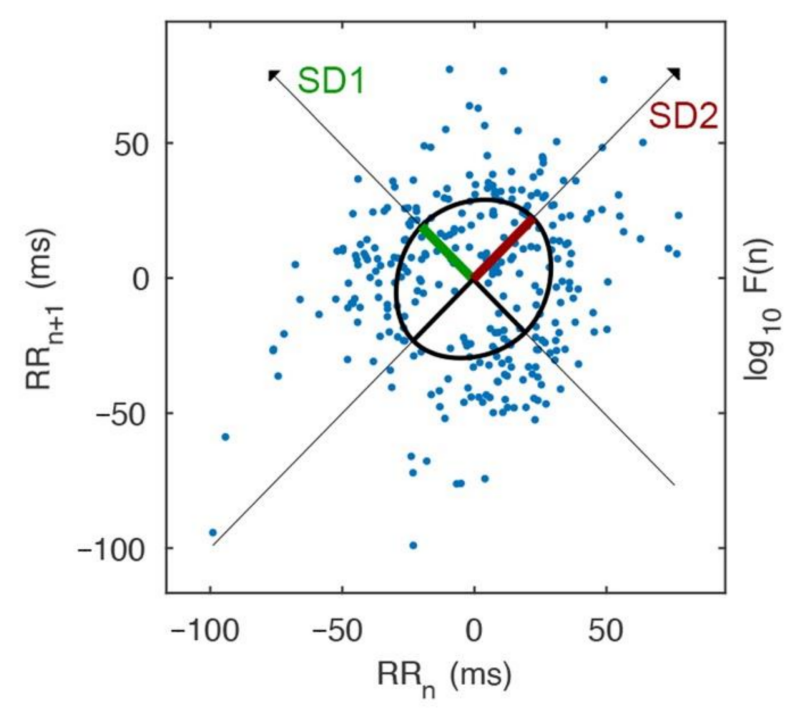

Females $(n=48)$

Proportion: SD1 $\approx$ SD2

Shape: Approximately rounded

Figure 2. Comparison of Poincaré plots in all ischemic stroke patients by age and sex. Poincaré plots of patients under 60 years old (A). Poincaré plots of patients over 60 years old (B). Poincaré plots of male patients (C). Poincaré plots of female patients (D). Note: Poincaré plots were presented as representative of each group.

After controlling for age and sex as shown in Table 3, there was statistical significance at $p<0.001$ with moderate and negative partial correlations between PNS and SD2 $(r=-0.47), \mathrm{SD} 2 / \mathrm{SD} 1(r=-0.47), \mathrm{LF}(r=-0.38)$, and LF/HF ratio $(r=-0.37)$. There was also a weak and negative partial correlation between PNS and DBP $(r=-0.24, p=0.012)$. There were moderate and positive partial correlations between PNS and SD1 $(r=0.47$, $p<0.001)$, HF $(r=0.38, p<0.001)$, and PTT $(r=0.29, p=0.002)$. In addition, there was statistical significance at $p<0.001$ with moderate and negative partial correlations between SNS and SD1 $(r=-0.39)$ and HF $(r=-0.28)$. There was also a weak and negative partial 
correlation between SNS and PTT $(r=-0.21, p=0.026)$. There were moderate and positive partial correlations between SNS and SD2/SD1 $(r=0.40, p<0.001)$, SD2 $(r=0.39, p<0.001)$, $\mathrm{LF} / \mathrm{HF}$ ratio $(r=0.29 p=0.002)$, DBP $(r=0.28 p=0.003)$, and $\operatorname{LF}(r=0.27, p=0.004)$. In contrast, there was no statistical significance between autonomic indices and SBP, PP, and MAP.

Table 3. A nonparametric partial correlation in all ischemic stroke patients with age and sex adjusted.

\begin{tabular}{ccccc}
\hline \multirow{2}{*}{ Variable (Unit) } & \multicolumn{2}{c}{ PNS Index } & \multicolumn{2}{c}{ SNS Index } \\
\cline { 2 - 5 } & Correlation $(r)$ & $p$ Value & Correlation $(r)$ & $p$ Value \\
\hline HRV indices & & & & \\
LF (n.u.) & -0.38 & $<0.001^{*}$ & 0.27 & $0.004^{*}$ \\
HF (n.u.) & 0.38 & $<0.001^{*}$ & -0.28 & $<0.001^{*}$ \\
LF/HF ratio & -0.37 & $<0.001^{*}$ & 0.29 & $0.002^{*}$ \\
SD1 (\%) & 0.47 & $<0.001^{*}$ & -0.39 & $<0.001^{*}$ \\
SD2 (\%) & -0.47 & $<0.001^{*}$ & 0.39 & $<0.001^{*}$ \\
SD2/SD1 & -0.47 & $<0.001^{*}$ & 0.40 & $<0.001^{*}$ \\
\hline PTT and BP & & & & \\
indices & & & -0.21 & $0.026^{*}$ \\
PTT (ms) & 0.29 & $0.002^{*}$ & 0.09 & 0.373 \\
SBP (mmHg) & -0.09 & 0.372 & 0.28 & $0.003^{*}$ \\
DBP (mmHg) & -0.24 & $0.012^{*}$ & -0.08 & 0.404 \\
PP (mmHg) & 0.04 & 0.679 & 0.18 & 0.066 \\
MAP (mmHg) & -0.17 & 0.086 & &
\end{tabular}

Note: ${ }^{*}$ denotes significance level at $p<0.05$.

\section{Discussion}

The present study aimed to explore the association between ANS and ischemic stroke through nonlinear HRV and PTT. The results indicated that ANS modulation among ischemic stroke patients differed in age and sex. Note that as there was no control group, this difference could simply be related to physiological differences in age and sex. Our results showed that older patients had a higher parasympathetic tone and PP, but a lower DBP than their younger counterparts. In addition, the SD1, SD2, and SD2/SD1 parameters differed between the younger and the older groups, and between men and women. Moreover, elliptical and circular Poincaré plot shapes characterized the young patients and older patients, respectively, while oval and approximately rounded plot shapes characterized the male patients and female patients, respectively. Furthermore, the PNS index was positively associated with HF, SD1 and PTT (negatively associated with the SNS), but negatively associated with LF, LF/HF ratio, SD2, SD2/SD1 and DBP (positively associated with the SNS).

The older patients had a higher parasympathetic tone than the younger patients. This result indicated that the ANS of older patients was more active within the PNS than in younger patients in balancing the SNS. This ANS dysfunction provided additional key information about ischemic stroke by suggesting that the difference between the elderly patients and the younger patients was not mainly involved with the low modulation of the SNS. It was rather due to an overactive PNS modulation. This could be explained by the DBP and PP results, which found that the elderly patients had a lower DBP and a higher PP than the younger patients. Regarding aging, low DBP caused by the loss of elasticity of arteries and high PP was affected by vasoconstriction. Together, these indicated that as blood flow to the heart and blood volume to the brain were lowered, then the PNS became more dominant.

The parameters of SD1, SD2, and SD2/SD1 were found to be useful indices in differentiating between the patients. The older patients had a higher SD1, but lower SD2 and SD2/SD1 than the younger patients; the same result was found when comparing the female patients to the male patients. This indicated that the older and female patients had a higher PNS response and a lower SNS response than the younger and male patients. It seemed 
that the older and female patients were confronted with the elevated PNS much more than the younger and male patients in controlling the stability of the ANS during the stroke event. This ANS pattern was also found in the circular and approximately rounded shapes of the Poincaré plots in the elderly and female patients, in which the PNS suppressed the SNS to the same level as indicating to the ischemic stroke. This evidence is supported by the mortality rate among ischemic stroke patients, as elderly females were at the highest risk [24]. Regarding the healthy participants, a previous study of short-term HRV found that there were no differences in SD1 and SD2 between males and females in both young and elderly groups [25].

Moreover, the results supported previous studies which found that the PNS and SNS were linked to HF and LF, respectively [26,27]. Additionally, the LF/HF ratio was linked to the balance between SNS and PNS [28]. The study suggested using the SD1 and PTT parameters for the PNS index, and the SD2 and SD2/SD1 parameters for the SNS index in ischemic strokes, after controlling for age and sex. Thus, the study recommended using the PNS parameters as a priority index for ischemic stroke.

The strengths of this study include that, to our knowledge, this is the first study that explores autonomic modulation through both linear and nonlinear HRV indices including PTT in Thai ischemic stroke patients. All assessments were noninvasive and performed by well-trained personnel using standardized protocol under standardized quality control systems. The presence of ischemic stroke in patients was verified by a full examination by the hospital, including the MRI scan. Nonetheless, there are some limitations to this study. First, HRV was performed using short-term recordings of $5 \mathrm{~min}$. Generally, a longer duration for short-term recordings of 15-30 $\mathrm{min}$ is preferred. A more robust assessment of HRV data could have yielded a more rigorous standard of the measurement. Second, it should be emphasized that our HRV data represent daytime, which may not reflect the overall activities of PNS and SNS that originate from $24 \mathrm{~h}$. Third, due to time limitations and access restrictions by the hospital, the assessment of cognitive performances was not possible and the level of missing data on the National Institutes of Health Stroke Scale (NIHSS) scores was high. Fourth, patients with diabetes, sleep apnea, and the use of cardio-selective drugs - all of which could have possible influence on their autonomic responses-were not excluded from the study. Fifth, the study did not collect personal or lifestyle information, such as smoking status, exercise patterns, occupation, or working hours, which could have added complementary information on autonomic control. This addresses the need for a large-scale prospective investigation of a 24-h Holter ECG for HRV measurements that include cognitive tests, NIHSS scores, sociodemographic information, clinical data, and related risk factors.

\section{Conclusions}

Among Thais who have experienced ischemic stroke, the PNS response was predominant in older and female patients while the SNS response was lower than in the younger and male patients. The older and female patients also showed in their Poincaré plots circular and approximately rounded shapes, respectively, with the SD1 radiuses close to the SD2 radiuses. These results suggested that there was an overactivity parasympathetic response to ischemic stroke in the elderly and female patients. Moreover, PNS response was positively associated with SD1 and PTT but negatively associated with SD2 and SD2/SD1, after controlling for age and sex, in all patients. Therefore, the study recommended using the SD1, SD2, SD2/SD1, and Poincaré plots including PTT as additional parameters in assessing autonomic modulation, particularly in the PNS, among ischemic stroke patients.

Author Contributions: Conceptualization and formal analysis, W.C.; Investigation, P.C.; Writingreview and editing, W.P. These authors contributed equally to this work. All authors have read and agreed to the published version of the manuscript.

Funding: The article publishing charge was partly funded by Chulalongkorn University. 
Institutional Review Board Statement: The study was conducted according to the guidelines of the Declaration of Helsinki, and approved by the Human Research Ethics Committee of Faculty of Medicine, Thammasat University (MTU-EC-IM-018154).

Informed Consent Statement: Informed consent was obtained from all patients involved in the study.

Data Availability Statement: The data are not publicly available due to the restrictions imposed by data providers and the ethical approval that governs the fieldwork. Access to data would only be granted upon request. For further inquiries, please contact the first author at warawoot.c@chula.ac.th.

Acknowledgments: This research is supported by the Ratchadapisek Somphot Fund for Postdoctoral Fellowship, Chulalongkorn University. W.P. is supported by Chulalongkorn University through the Population, Family Dynamics, and Social Policy Research Unit.

Conflicts of Interest: The authors declare no conflict of interest.

\section{References}

1. Kongbunkiat, K.; Kasemsap, N.; Thepsuthammarat, K.; Tiamkao, S.; Sawanyawisuth, K. National Data on Stroke Outcomes in Thailand. J. Clin. Neurosci. 2015, 22, 493-497. [CrossRef]

2. Suwanwela, N.C. Stroke Epidemiology in Thailand. J. Stroke 2014, 16, 1-7. [CrossRef]

3. Zhao, M.; Guan, L.; Wang, Y. The Association of Autonomic Nervous System Function with Ischemic Stroke, and Treatment Strategies. Front. Neurol. 2020, 10, 1411. [CrossRef]

4. Battaglini, D.; Robba, C.; Lopes da Silva, A.; dos Santos Samary, C.; Leme Silva, P.; Dal Pizzol, F.; Pelosi, P.; Rocco, P.R. Brain-heart Interaction after Acute Ischemic Stroke. Crit. Care 2020, 24, 163. [CrossRef]

5. Lees, T.; Shad-Kaneez, F.; Simpson, A.M.; Nassif, N.T.; Lin, Y.; Lal, S. Heart Rate Variability as a Biomarker for Predicting Stroke, Post-stroke Complications and Functionality. Biomark Insights 2018, 13, 1-13. [CrossRef] [PubMed]

6. Acharya, U.R.; Joseph, K.P.; Kannathal, N.; Lim, C.M.; Suri, J.S. Heart Rate Variability: A Review. Med. Biol. Eng. Comput. 2006, 44, 1031-1051. [CrossRef] [PubMed]

7. Chuangchai, W.; Pothisiri, W. Postural Changes on Heart Rate Variability among Older Population: A Preliminary Study. Curr. Gerontol. Geriatr. Res. 2021, 2021, id6611479. [CrossRef]

8. Shaffer, F.; Ginsberg, J.P. An overview of heart rate variability metrics and norms. Front. Public Health 2017, 5, 258. [CrossRef]

9. Adjei, T.; von Rosenberg, W.; Nakamura, T.; Chanwimalueang, T.; Mandic, D.P. The ClassA Framework: HRV Based Assessment of SNS and PNS Dynamics Without LF-HF Controversies. Front. Physiol. 2019, 10, 505. [CrossRef]

10. Constantinescu, V.; Matei, D.; Costache, V.; Cuciureanu, D.; Arsenescu-Georgescu, C. Linear and Nonlinear Parameters of Heart Rate Variability in Ischemic Stroke Patients. Neurol. I Neurochir. Pol. 2018, 52, 194-206. [CrossRef] [PubMed]

11. de Souza Filho, L.F.M.; de Oliveira, J.C.M.; Ribeiro, M.K.A.; Moura, M.C.; Fernandes, N.D.; de Sousa, R.D.; Pedrino, G.R.; Rebelo, A.C.S. Evaluation of the Autonomic Nervous System by Analysis of Heart Rate Variability in the Preterm Infants. BMC Cardiovasc. Disord. 2019, 19, 198. [CrossRef]

12. Chuangchai, W. Pulse Transit Time in Ageing as Early Biomarker for Risk of Dementia. Thai J. Ergon. 2020, 3, 35-44.

13. Rangsungnoen, S.; Chanbenjapipu, P.; Mathuradavong, N.; Suwanprasert, K. A Hybrid Signal Processing of RR Intervals from QTc Variation Searching Arrhythmia and Improving Heart Rate Variability Assessment in Acute Large Artery Ischemic Stroke. J. Med. Eng. 2016, 2016, 9823026. [CrossRef] [PubMed]

14. Suwanprasert, K.; Phumdecha, C.; Muengtaweepongsa, S. Neurovascular Oxidative Stress and Autonomic Open Access Modulation Contributing to QT Interval Variations in Acute Large Artery Ischemic Stroke. Austin J. Cerebrovasc. Dis. Stroke 2017, 4 , id1071.

15. Kannakorn, I.; Kesorn, S.; Sombat, M. Correlation between Decreased Parasympathetic Activity and Reduced Cerebrovascular Reactivity in Patients with Lacunar Infarct. Curr. Neurovasc. Res. 2017, 14, 65-70.

16. Chondaen, N.; Plusiri, P.; Srisawad, S.; Meesribau, S.; Isariyapan, O.; Chotimol, P.; Roongpiboonsopit, D.; Sangthong, B.; Chinda, K.; Srisoparb, W. Relationships between Heart Rate Variability, Motor Impairments and Level of Disability in Chronic Ischemic Stroke Patients. Srinagarind Med. J. 2020, 35, 746-752.

17. Hanchaiphiboolkul, S.; Poungvarin, N.; Nidhinandana, S.; Suwanwela, N.C.; Puthkhao, P.; Towanabut, S.; Tantirittisak, T.; Suwantamee, J.; Samsen, M. Prevalence of stroke and stroke risk factors in Thailand: Thai Epidemiologic Stroke (TES) Study. J. Med. Assoc. Thail. 2011, 94, 427-436.

18. Hanchaiphiboolkul, S.; Puthkhao, P.; Towanabut, S.; Tantirittisak, T.; Wangphonphatthanasiri, K.; Termglinchan, T.; Nidhinandana, S.; Suwanwela, N.C.; Poungvarin, N. Factors Predicting High Estimated 10-Year Stroke Risk: Thai Epidemiologic Stroke Study. J. Stroke Cerebrovasc. Dis. 2014, 23, 1969-1974. [CrossRef]

19. Hanchaiphiboolkul, S.; Suwanwela, N.C.; Poungvarin, N.; Nidhinandana, S.; Puthkhao, P.; Towanabut, S.; Tantirittisak, T.; Suwantamee, J.; Samsen, M. Risk of Metabolic Syndrome for Stroke Is Not Greater than the Sum of its Components: Thai Epidemiologic Stroke (TES) Study. J. Stroke Cerebrovasc. Dis. 2013, 22, e264-e270. [CrossRef]

20. Pipatvanichgul, B.; Hanchaiphiboolkul, S.; Puthkhao, P.; Tantirittisak, T.; Towanabut, S. Association between Socioeconomic Status and Major Risk Factors of Stroke: Thai Epidemiologic Stroke (TES) Study. J. Med. Assoc. Thail. 2015, 98, 739-747. 
21. DeMers, D.; Wachs, D. Physiology, Mean Arterial Pressure; StatPearls Publishing LLC.: Treasure Island, FL, USA, 2020.

22. Tarvainen, M.P.; Niskanen, J.P.; Lipponen, J.A.; Ranta-Aho, P.O.; Karjalainen, P.A. Kubios HRV—Heart Rate Variability Analysis Software. Comput. Methods Programs Biomed. 2014, 113, 210-220. [CrossRef]

23. Fritz, C.O.; Morris, P.E.; Richler, J.J. Effect Size Estimates: Current Use, Calculations, and Interpretation. J. Exp. Psychol. Gen. 2012, 141, 2-18. [CrossRef]

24. Phan, H.T.; Blizzard, C.L.; Reeves, M.J.; Thrift, A.G.; Cadilhac, D.; Sturm, J.; Heeley, E.; Otahal, P.; Konstantinos, V.; Anderson, C.; et al. Sex Differences in Long-Term Mortality After Stroke in the INSTRUCT (INternational STRoke oUtComes sTudy). Circ. Cardiovasc. Q. Outcomes 2017, 10, e003436. [CrossRef]

25. Voss, A.; Schroeder, R.; Heitmann, A.; Peters, A.; Perz, S. Short-Term Heart Rate Variability-Influence of Gender and Age in Healthy Subjects. PLoS ONE 2015, 10, e0118308. [CrossRef] [PubMed]

26. Tobaldini, E.; Sacco, R.M.; Serafino, S.; Tassi, M.; Gallone, G.; Solbiati, M.; Costantino, G.; Montano, N.; Torgano, G. Cardiac Autonomic Derangement is Associated with Worse Neurological Outcome in the Very Early Phases of Ischemic Stroke. J. Clin. Med. 2019, 8, 852. [CrossRef]

27. Verma, A.K.; Aarotale, P.N.; Dehkordi, P.; Lou, J.S.; Tavakolian, K. Relationship between Ischemic Stroke and Pulse Rate Variability as a Surrogate of Heart Rate Variability. Brain Sci. 2019, 9, 162. [CrossRef]

28. Shibasaki, K.; Yamada, S.; Ouchi, Y.; Akishita, M.; Ogawa, S. Effect of Rehabilitation on Recovery of Sympathetic Nervous Activity Measured According to Heart Rate Variability in Frail Elderly Adults. J. Am. Geriatr. Soc. 2016, 64, e15-e16. [CrossRef] [PubMed] 